\title{
STUDY ON THE RAPID PLANNING OF TRAFFIC ROUTES BASED ON MAP MATCHING ALGORITHM
}

\author{
Wenjun Zhu' ${ }^{1}$, Huisheng Shi \\ ${ }^{1,2}$ Henan College of Surveying and Mapping, Zhengzhou 450000, Henan, China \\ ${ }^{1}$ Department of Space Information Engineering, \\ ${ }^{2}$ Department of Remote Sensing Engineering, \\ E-mail: shuimeilu3@163.com
}

\begin{abstract}
With the development of positioning system, the data of vehicle trajectory that can be obtained by the traffic department is more and more huge, and it is more difficult to use the data of vehicle trajectory points and map to plan the traffic route. In order to improve the efficiency of traffic route planning, this paper briefly introduced the map matching algorithm for traffic route planning and improves the part of the optimal path selection in the traditional map matching algorithm by using Bayes theorem. The simulation results of the two algorithms showed that the trend of the two algorithms was basically the same, but the path of the improved algorithm was smoother, and the traditional algorithm had different degrees of distortion in the dense road network; the time interval of the track points provided by the positioning system affected the accuracy of the traffic route planning, the greater the interval, the lower the accuracy of the two algorithms, but under the same time interval, the accuracy of the improved algorithm was always higher than that of the traditional algorithm; the improved algorithm needed less operation time and had higher efficiency in the planning of traffic route than the traditional algorithm.
\end{abstract}

Keywords: Map Matching Algorithm, Traffic Route, Bayes Theorem, Error Circle Search.

\section{Introduction}

The development of positioning technology and the popularization of vehicle positioning device greatly facilitate people's travel. With the help of the map in the vehicle positioning system and navigation software, the vehicle driver can drive in a relatively unfamiliar area, which can also largely avoid the situation of getting lost [1]. In addition, even if the driver drives in a familiar road section, with the help of positioning system and navigation software, the optimal path can be quickly planned to avoid road congestion as much as possible. In addition to personal use, with the help of vehicle positioning system and Internet of things, the transportation department can also plan a wide range of traffic routes reasonably and quickly, so as to reduce the frequency of congestion and improve the quality of public transport services [2].The vehicle positioning system can obtain the change of vehicle position during driving. Theoretically, the traffic planning route of a vehicle can be obtained as long as the location points are arranged in chronological order and the map is overlapped. However, the above method is only applicable to high-precision positioning system, or error free positioning. There must be errors in the positioning system in practical application. If the vehicle traffic route planning is carried out completely according to the time sequence, the route planning will not conform to the actual road situation, which will affect the traffic [3]. Therefore, in order to connect the vehicle trajectory with the road network and obtain the accurate traffic planning route, map matching algorithm is essential. Schweizer et al. [4] proposed a map matching algorithm and applied it to the route planning of bicycle GPS track and verified that the algorithm could well tolerate the lack of road network information and can achieve reliable and fast map matching even in dense street network and interrupted GPS data flow. Tang et al. [5] proposed an improved hierarchical fuzzy reasoning method based on c-measure map matching algorithm, in which c-measure represents the certainty or probability of vehicle driving on the actual road, and matched the vehicle trajectory with the shape curve of the candidate road to plan the vehicle's traffic route using the historical positioning information. Finally, four different paths in Beijing were taken as examples, and the effectiveness and superiority of this method were verified. Singh et al. [6] collected $47 \mathrm{~km}$ of track route data set, compared the map matching algorithms based on geometry, topology and Kalman filter, and found that the performance of the map matching algorithm based on Kalman filter was significantly better than the other two algorithms. In this study, the map matching algorithm for traffic route planning was briefly 
introduced, the part of the optimal path selection in the traditional map matching algorithm was improved by using Bayes theorem, and finally the two algorithms were simulated.

\section{Map Matching Algorithm}

\section{- Traditional map matching algorithm}

The traditional map matching algorithm is based on the geometric characteristics of the road network and vehicle positioning trajectory points, and the corresponding vehicle traffic planning route can be obtained by allocating the trajectory points to the road network according to the shortest distance principle. However, with the expansion of the road network, the sampling frequency of ordinary vehicle positioning system is low, which makes the accuracy of map matching and traffic route planning decrease. Therefore, it is necessary to improve the performance of map matching algorithm, so as to improve the accuracy of traffic route rapid planning.

The map matching algorithm is generally divided into two steps: (1) search the candidate planning road sections according to the track points and road network; (2) select the best planning road section from the candidate planning road sections according to certain rules.

Firstly, in the map matching algorithm, road network [7] is expressed as:

$$
G=(L, P)
$$

where $G$ stands for the whole road network, $L$ is a set of road sections (each road section contains two attributes: spatial and non-spatial, the spatial attributes are the start and end points and internal nodes of the sections, and the non-spatial attributes include information such as section number, name, length and type), and $P$ is a set of end points of road section. End points of a road section are generally road intersections or vehicle collection points. In this study, road intersections are selected as the end points of road section. Each end point includes the end point number, name, longitude and latitude coordinates, and the set of road sections starting from or ending at that end point. The vehicle track points [8] are expressed as:

$$
\left\{\begin{array}{l}
G P=\left\{g p_{i}\right\} \\
m G P=\left\{m g p_{i}\right\}
\end{array}\right.
$$

where GP stands for a set of unmatched track points, $m G P$ stands for the set of matched track points, $g p_{i}$ stands for the i-th unmatched track point, which contains the information of the vehicle such as ID, latitude and longitude coordinates, sampling time point, speed, direction angle, etc, $m g p_{i}$ is the i-th matched track point, which not only includes the information types of $g p_{i}$, but also includes information of matched road section.

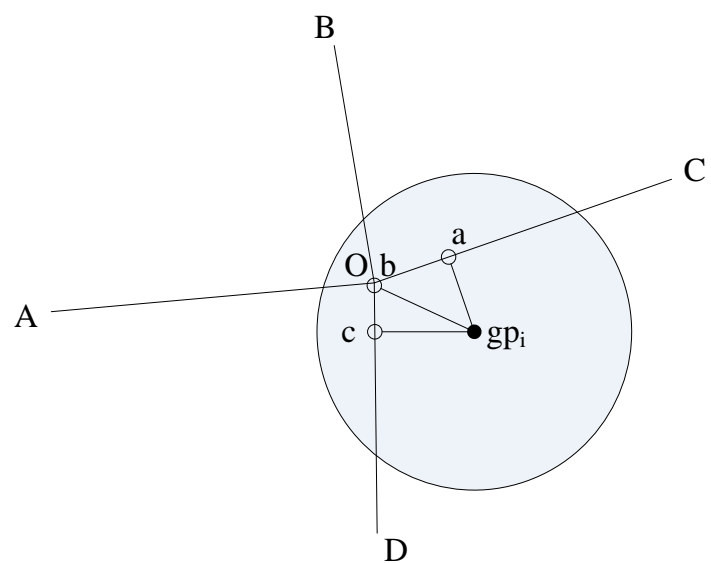

Figure 1: Searching of candidate planning road sections

After the road network and vehicle trajectory network are expressed in the above way, the traffic route is planned using the map matching algorithm according to the collected vehicle trajectory points. Firstly, the candidate road sections are searched according to the vehicle track points. In this study, the candidate road sections are searched using the error circle search method. As shown in Figure 1, black dot $g p_{i}$ is the track point to be matched, and roads within the error circle which takes $g p_{i}$ as the center of circle and $r$ as the radius are the candidate roads. Error circle radius $r$ is determined by the positioning error radius and road simplification error. As can be seen in Figure 1, there are four roads $(\mathrm{OA}, \mathrm{OB}, \mathrm{OC}, \mathrm{OD})$ within the circle which takes $g p_{i}$ as the center of circle and $r$ as radius, all of which are candidate roads [9]. Then the candidate points for correcting the track points are selected from each candidate road. The selection method of the candidate points is: make a vertical line to the candidate road through $g p_{i}$, and determine the candidate points according to the vertical foot; when the vertical foot is in the candidate road, it is the candidate point, and when the vertical foot is outside the candidate road, the end point of the candidate road which is the nearest to $g p_{i}$ is selected as the candidate point. The duplicate points are deleted after selection of candidate point. In Figure 1, a and c are the candidate points of $\mathrm{OC}$ and OD respectively, and $b$ is the candidate point of $\mathrm{OA}$ and $\mathrm{OB}$.

After searching candidate roads and candidate points, the second step of the map matching algorithm starts, i.e., to select the optimal path. The optimal path is selected according to the rules from the set of candidate roads, and the trajectory points are corrected through the candidate points in the optimal path. 
The above steps are repeated to correct the set of vehicle trajectory points, and finally the traffic route planning map of the optimal path is obtained. The traditional method of selecting the optimal path is based on the principle of the shortest path. As the track route of vehicles is continuous, when selecting the optimal path, in addition to following the shortest path principle, the optimal path also needs to meet the network topology; when using candidate points in the optimal path to correct the track points, if there are multiple candidate points in the path, the candidate points with the shortest distance from the track points are selected as the correction points.

\section{- The improved map matching algorithm}

As mentioned above, the traditional map matching algorithm adopts the shortest path principle to select the optimal path on the premise of following the topological structure of the road network, but this selection method does not fully consider the positioning error and is relatively isolated when analyzing the track points, as it does not fully consider the front and back sequence connection in time. In this study, Bayesian theorem is used to select the optimal path in the candidate path set. The traffic route planning flow of the improved map matching algorithm is shown in Figure 2.

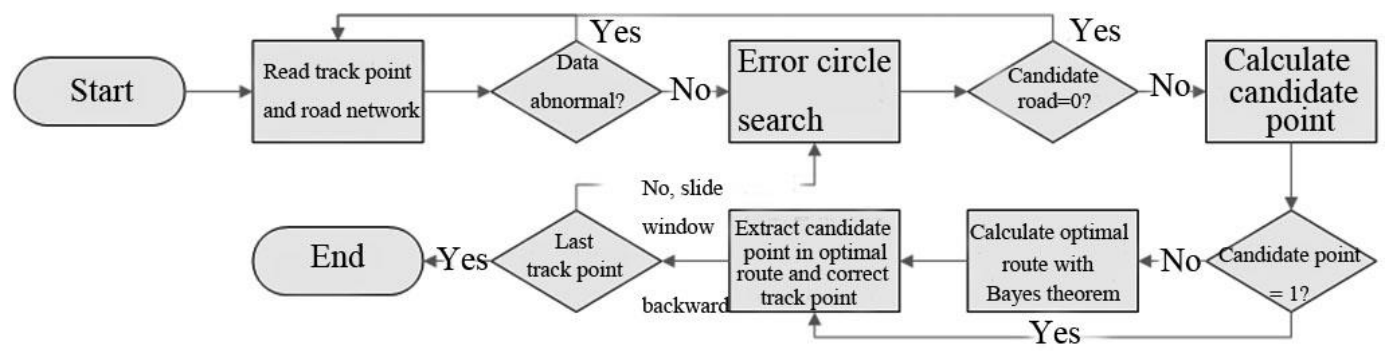

Figure 2: The traffic route planning process of the improved map matching algorithm

In this study, the Bayes theorem [10] for optimal path selection is a method of probability reasoning, which makes full use of previous prediction experience through prior distribution probability and conditional probability. It not only judges the characteristic trend of probability reasoning object, but also simplifies the association between objects. Its basic formula is as follows:

$$
g(\theta \mid x)=\frac{f(x \mid \theta) g(\theta)}{P(x)}
$$

where $g(\theta \mid x)$ stands for the inference probability of the object, $f(x \mid \theta)$ is the conditional probability [11], $g(\theta)$ is the prior distribution probability [12], and $P(x)$ is the total probability distribution of the sample set. The traffic route planning process improved by Bayes theorem is as follows:

(1) The collected data of vehicle positioning track points and the simplified road network are input, then the vehicle track points are read in turn according to the time sequence to determine whether the track is abnormal. If not, the next step proceeds; if there is any abnormal, the track point data are read again.

(2) The candidate road of the current track point is searched by using the error circle. If the candidate road cannot be found, the abnormality is recorded and the data are read again. After searching the candidate road, the candidate point is calculated according to the above method.

(3) If there is only one candidate point for the current track point, it can be directly used as the correction point for the track point; if there is more than one candidate point, the next step of optimal path selection is required.

(4) The optimal path selection model in this study relies on Bayes theorem, so the model meets the following assumptions: the time interval between the track points is short; in the track point sequence, there is a conditional probability relationship between the track points to be calculated and the track points before and after; the number of track points exceeds 5 . The size of the sliding window is 3 , and the probability is calculated by using the track points with a time interval of $m$ before and after the current track point. The calculation formula [13] is as follows:

$$
\left\{\begin{array}{l}
f_{s n}=f_{s t} \cdot f_{d i r} \\
f_{s t}=\frac{p\left(g p_{i-m}, g p_{i+m} \mid e_{k}\right) \cdot p\left(e_{k} \mid g p_{i}\right)}{p\left(g p_{i-m}, g p_{i+m}\right)} \\
f_{d i r}=\max \left\{e^{\frac{-\left|d i_{s n}-d i r_{e k}^{+}\right|}{180}}, e^{\frac{-\left|d i_{s n}-d i r_{e k}^{-}\right|}{180}}\right\}
\end{array}\right.
$$

where $f_{s n}$ is the probability that the candidate road section is optimal, $f_{s t}$ stands for the correlation probability between the track points to be matched and the track points before and after the time sequence [14], $f_{d i r}$ is azimuth probability, $p\left(g p_{i-m}, g p_{i+m} \mid e_{k}\right)$ is the probability that the track points before and after the time sequence of the track point to be matched belonging to candidate 
road section $e_{k}, p\left(e_{k} \mid g p_{i}\right)$ stands for the probability of $e_{k}$ appearing in track point, following the distribution of $p\left(e_{k} \mid g p_{i}\right): U(0,1), p\left(g p_{i-m}, g p_{i+m}\right)$ is the distribution of the track point before and after in all the samples, $d i r_{s n}$ is the azimuth angle, and $d i r_{e k}^{+}, d i r_{e k}^{-}$are the up and down directions of the candidate road section.

Through the above formula, the probability of all candidate road sections of the track points to be matched is calculated, and the candidate road section with the highest probability is selected as the optimal one.

5) After selecting the optimal road section, the candidate points in the road section are taken as the correction points of the track points to correct the track points.

(6) After the track point is corrected, whether the track point is the last track point is determined. If not, the process stops and the results are output.

If not, the window slides down to the next track point, and steps (2) $\sim$ are repeated.

\section{Example Analysis}

\section{- Experimental environment}

The traditional map matching algorithm and improved map matching algorithm mentioned above were compiled using $\mathrm{C}++$ language [15].

The experiment was carried out on a laboratory server with configurations of Windows 7 system, I7 processor and 16 G memory.

\section{- Experimental data}

As shown in Figure 3, an area of Zhengzhou city was taken as the subject, and the location data of taxis in this area was collected as the data set of traffic route planning for map matching.

The data set contained the track sequence of 1500 taxis, with a total of 330, 000 track points.

The collection time of the data set was from March 1, 2019 to March 7, 2019.

The time interval between the two adjacent track points in the track sequence of each vehicle was at least $10 \mathrm{~s}$.

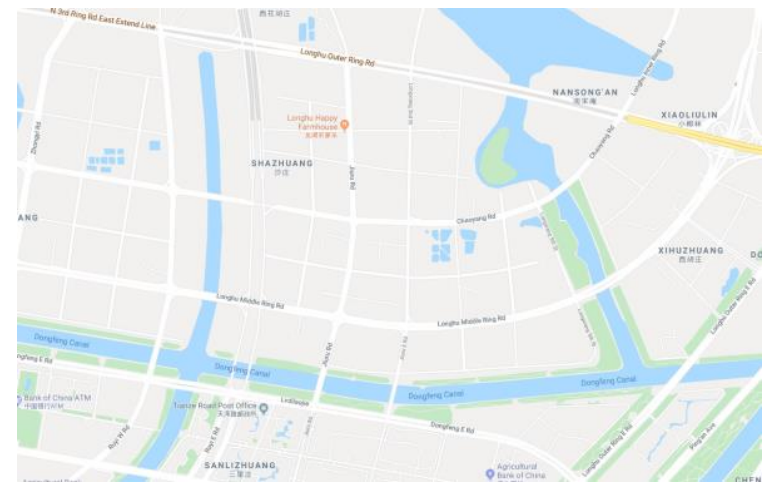

Figure 3: The map of some areas of Zhengzhou city

\section{- Experimental method}

Before using the map matching algorithm to plan the traffic route, the track sequence was classified according to the vehicle ID to reduce the calculation.

Then, the track points were extracted from the track samples of each taxi at intervals of $10 \mathrm{~s}, 15 \mathrm{~s}$, $20 \mathrm{~s}, 25 \mathrm{~s}$ and $30 \mathrm{~s}$ to form the track point data sets with different time intervals. Next, the track points of 30 taxis were randomly selected from the track point data sets of each time interval, and 400 track points were selected for each taxi, respectively. Finally the traffic route was planned using the traditional map matching method and improved map matching method.

\section{- Experimental results}

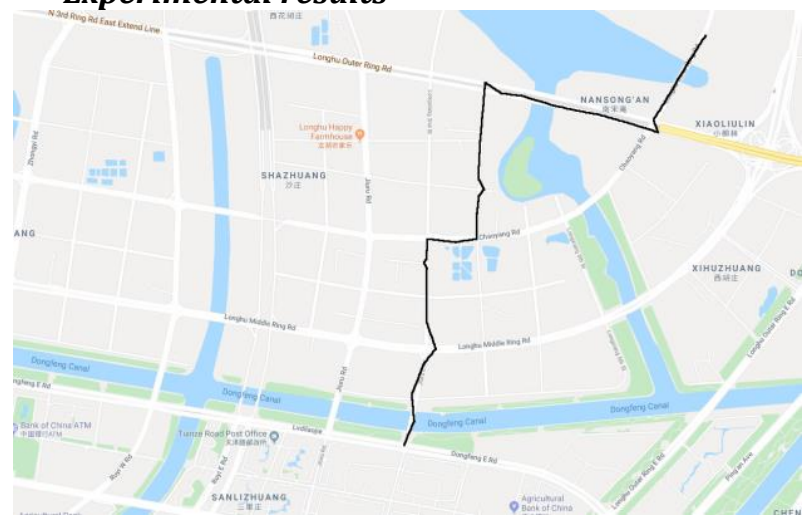

Figure 4: The traffic planning route based on the traditional map matching algorithm

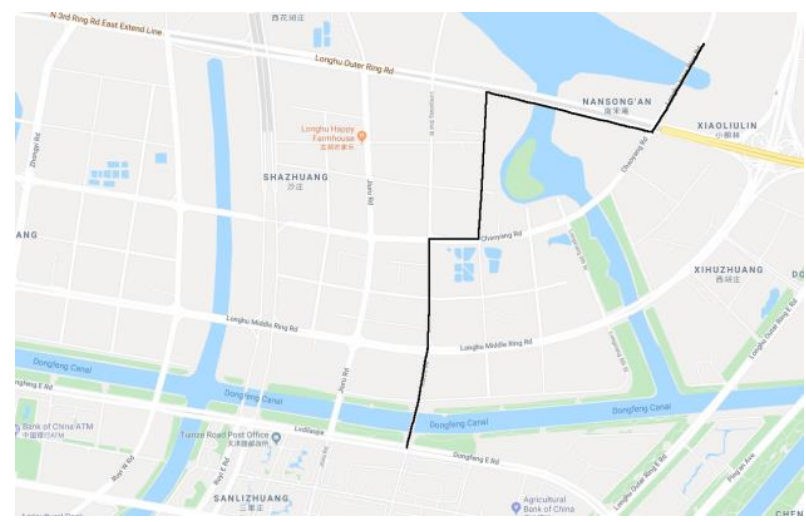

Figure 5: The traffic planning route based on the improved map matching algorithm

Limited by the space, this paper only shows the traffic planning route of a taxi's track points with 10 s time interval under two map matching algorithms, as shown in Figure 4 and 5 . It can be seen intuitively from Figure 4 and 5 that the trend of the traffic planning route calculated by the two map matching algorithms was basically the same under the same track point data, but the traffic planning route planned by the improved map matching algorithm was smoother, and the traffic planning route obtained by the traditional map matching algorithm was distorted in different degrees at some intersections. Through observation, it was found that 
the traditional map matching algorithm matched the track points to the adjacent road sections at intersections, eventually leading to the distortion of the whole line.

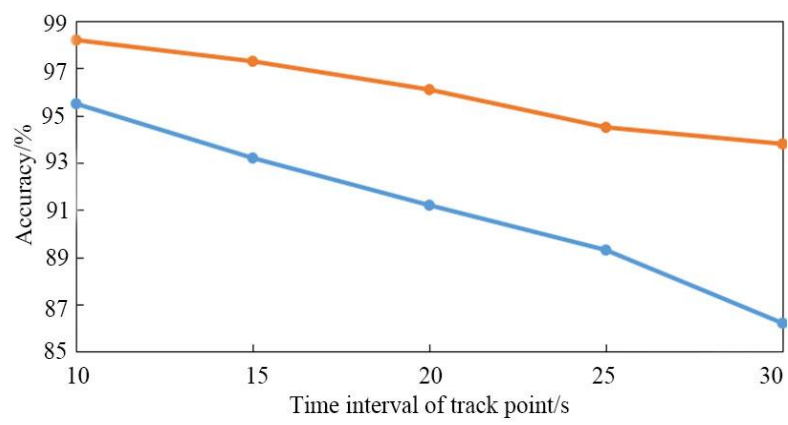

$\rightarrow-$ Traditional map matching algorithm $\rightarrow$ Improved map matching algorithm

Figure 6: Accuracy of two algorithms for traffic planning under different time intervals of track points

As shown in Figure 6, the accuracy of the traditional map matching algorithm was $95.5 \%$ and the accuracy of the improved map matching algorithm was $98.2 \%$ when the time interval of adjacent track points was $10 \mathrm{~s}$; the accuracy of the traditional map matching algorithm was $93.2 \%$ and the accuracy of the improved map matching algorithm was $97.3 \%$ when the time interval of adjacent track points was $15 \mathrm{~s}$; the accuracy of the traditional map matching algorithm was $91.2 \%$ and the accuracy of the improved map matching algorithm was $96.1 \%$ when the time interval of adjacent track points was $20 \mathrm{~s}$; the accuracy of the traditional map matching algorithm was $89.3 \%$ and the accuracy of the improved map matching algorithm was $94.5 \%$ when the time interval between adjacent tracks was $25 \mathrm{~s}$; the accuracy of the traditional map matching algorithm was $86.2 \%$ and the accuracy of the improved map matching algorithm was $93.8 \%$ when the time interval between adjacent tracks was $30 \mathrm{~s}$. It can be seen from Figure 6 that the accuracy of the traffic planning route obtained by the traditional or improved map matching algorithm decreased with the increase of the time interval of the track points used in the map matching algorithm.

The reason was as follows.

When the time interval of the track points was small, the moving distance of vehicles in space was small, and the relationship between space before and after was strong; therefore both the topological principle of the shortest route and the probability inference of Bayes theorem could accurately select the optimal route.

When the time interval increased, the moving distance of vehicles increased, and the relationship between space before and after was weak, and moreover the optimal road sections increased, which improved complexity and decreased accuracy.
Under the same time interval, the accuracy of the improved map matching algorithm was higher.

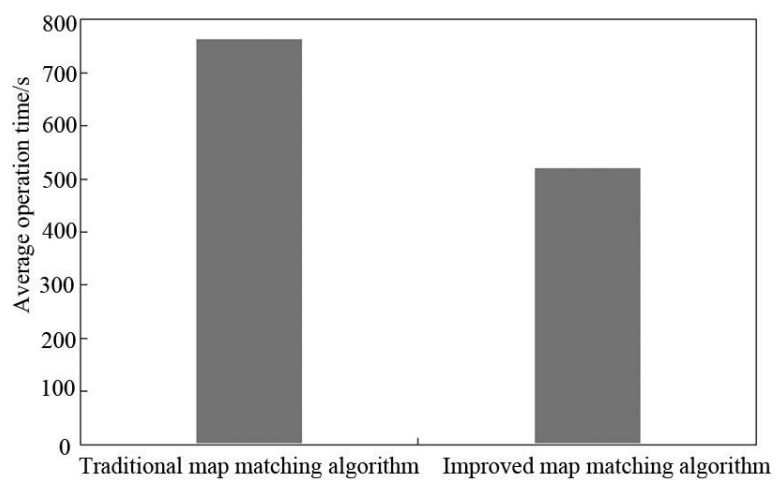

Figure 7: The average time of calculation of the traffic planning route by the two algorithms based on the track points of every vehicle

As shown in Figure 7, the average calculation time of the traditional map matching algorithm for the traffic planning route of every taxi was $763 \mathrm{~s}$, and that of the improved map matching algorithm was 521 s. It was seen from Figure 7 that the improved map matching algorithm was more efficient than the traditional map matching algorithm.

The reason was that the traditional map matching algorithm used the shortest route principle when selecting the optimal path and also considered the topological structure of the overall path, which was relatively large in calculation, but the improved map matching algorithm used Bayes theorem to link some track segments for probability reasoning when selecting the optimal path, and the calculation amount greatly reduced as the calculation of the whole route was not needed.

\section{Conclusion}

This paper briefly introduced the map matching algorithm for traffic route planning and improved the part of selection of optimal route in the traditional map matching algorithm to improve the efficiency of traffic route planning.

The optimal route was selected by the probabilistic reasoning method of Bayes theorem. Then the simulation experiment was carried out on the traditional and improved map matching algorithms, and the results are as follows.

The trend of the traffic planning route calculated by the two map matching algorithms was basically the same, but the traditional traffic planning route had different degrees of distortion in the complex road network area, and the improved traffic planning route was smoother.

With the increase of the time interval of track points used for calculating traffic route, the accuracy of the traffic planning route gradually decreased, but the accuracy of the improved algorithm was always 
higher than the traditional algorithm under the same time interval of track points.

In the aspect of planning efficiency of the traffic route, the average time needed by the improved map matching algorithm was shorter.

\section{References}

[1] Romon S, Bressaud X, Lassarre S, Pierre G S, Khoudour L. "Map-matching Algorithm for Large Databases," The Journal of Navigation, 2015, 68(5):971-988.

[2] Ling Y, Dan L, Song H. "A map-matching algorithm with low-frequency floating car data based on matching path," EURASIP Journal on Wireless Communications and Networking, 2018, 2018(1):146.

[3] Kim J, Mahmassani H S. "Spatial and temporal characterization of travel patterns in a traffic network using vehicle trajectories," Transportation Research Part C: Emerging Technologies, 2015:S0968090X15002508.

[4] Rupi F, Bernardi S, Schweizer J. "Map-matching algorithm applied to bicycle global positioning system traces in Bologna," IET Intelligent Transport Systems, 2016, 10(4):244-250.

[5] Tang J, Zhang S, Zou Y, Liu F. "An adaptive mapmatching algorithm based on hierarchical fuzzy system from vehicular GPS data," PLoS ONE, 2017, 12(12):e0188796.

[6] Singh J, Singh S, Singh S, Singh H. "Evaluating the performance of map matching algorithms for navigation systems: an empirical study," Spatial Information Research, 2018, 27(1):6374.

[7] Liu Y, Li Z. "Erratum to: A novel algorithm of low sampling rate GPS trajectories on mapmatching," Eurasip Journal on Wireless Communications \& Networking, 2017, 2017(1):150.
[8] Taneja S, Akinci B, Garrett J H, Soibelman L. "Algorithms for automated generation of navigation models from building information models to support indoor map-matching," Automation in Construction, 2016, 61:24-41.

[9] Taguchi S, Koide S, Yoshimura T. "Online Map Matching With Route Prediction," IEEE Transactions on Intelligent Transportation Systems, 2018, PP(99):1-10.

[10] Castillo I, Schmidthieber J, Vaart A V D. "Bayesian linear regression with sparse priors," Annals of Statistics, 2015, 43(5):1986-2018.

[11] Feng T, Timmermans H J P. "Detecting Spatial and Temporal Route Information of GPS Traces," Lecture Notes in Geoinformation \& Cartography, 2015:61-75.

[12] Si L, Wang Z, Liu X, Tan C, Xu J, Zheng K. "MultiSensor Data Fusion Identification for Shearer Cutting Conditions Based on Parallel QuasiNewton Neural Networks and the DempsterShafer Theory," Sensors, 2015, 15(11):2877228795.

[13] Jagadeesh G R, Srikanthan T. "Online MapMatching of Noisy and Sparse Location Data With Hidden Markov and Route Choice Models," IEEE Transactions on Intelligent Transportation Systems, 2017, 18(9):2423-2434.

[14] Rajabi M M, Ataie-Ashtiani B. "Efficient fuzzy Bayesian inference algorithms for incorporating expert knowledge in parameter estimation," Journal of Hydrology, 2016, 536:255-272.

[15] Sobreira H, Costa C M, Sousa I, Rocha LF, Lima J, Farias P, Costa P J C, Moreira A P. "MapMatching Algorithms for Robot SelfLocalization: A Comparison Between Perfect Match, Iterative Closest Point and Normal Distributions Transform," Journal of Intelligent \& Robotic Systems, 2018(2):1-14. 